\title{
The role of diet in rheumatoid arthritis
}

\section{Marta Skoczyńska, Jerzy Świerkot}

Department and Clinic of Rheumatology and Internal Medicine, Wrocław Medical Hospital, Wrocław Medical University, Poland

\begin{abstract}
Rheumatoid arthritis (RA) is a systemic connective tissue disease which develops in the course of an autoimmune inflammatory process triggered by environmental factors in a genetically predisposed person. One of the environmental factors is the diet. RA patients' adherence to a healthy diet remains low, despite plentiful data confirming positive effects of some foods, e.g. fish rich in n-3 polyunsaturated fatty acids (PUFAs), as well as the negative influence of unhealthy eating patterns, such as high consumption of fats and sugars, on RA incidence, activity and treatment response. In this review, we present current knowledge on the role of diet in rheumatoid arthritis, including dietary factors' preventive/promoting influence on RA development, as well as their impact on RA activity. We hope this article will aid and encourage clinicians to recommend a relevant dietary intervention to their RA patients.
\end{abstract}

Key words: diet, disease activity, rheumatoid arthritis.

\section{Introduction}

Rheumatoid arthritis (RA) is a systemic connective tissue disease affecting predominantly joints. The disease develops in the course of an autoimmune inflammatory process triggered by environmental factors in a genetically predisposed person. One such environmental factor, which may either increase or decrease the risk of RA incidence, as well as having an immunomodulatory (exacerbating or attenuating) influence on disease activity, is the diet.

\section{Dietary protein components potentially triggering rheumatoid arthritis}

It has been well documented that Porphyromonas gingivalis bacteria, colonizing the oral cavity, may produce an enzyme that citrullinates proteins, which trigger anti-citrullinated protein antibody (ACPA) production. Tsuda et al. [1], investigating the role of diet in the pathogenesis of RA, confirmed the presence of cross-reactivity between ACPA obtained from RA patients' serum and citrullinated proteins derived from viruses (EBNA-1), bacteria (Micrococcus luteus, Mycobacterium gastri, Nocardia brasiliensis), fungi (Aspergillus fumigatus, Candida albicans, Cryptococcus neoformans) and plants (Oryza sativa, Solanum lycopersicum, Glycine max). They concluded that eating foods containing the aforementioned antigens in persons colonized with $P$. gingivalis may trigger ACPA production, subsequently inducing RA through cross-reactivity reactions with tissue antigens.

Since it has been discovered that plant-derived dietary lectins may be involved in the pathogenesis of some inflammatory diseases, including RA, Gong et al. studied molecular mechanisms behind the lectin-induced inflammatory process. They found that plant lectins can trigger caspase- 1 activation and IL-1 $\beta$ secretion via the NLRP3 (NACHT, LRR and PYD domains-containing protein 1) inflammasome in vivo. Therefore it may be concluded that lectins, present in significant quantities in plant seeds, have a potential of becoming a "danger signal" activating the NLRP3 inflammasome and thus promoting the inflammatory process [2].

\section{The role of microbiota in rheumatoid arthritis}

Rheumatoid arthritis and celiac disease (CD) are both autoimmunologic disorders and often share similar rheumatic as well as gastrointestinal symptoms. Recently, there have been efforts to find pathophysiological links between them. Both diseases, despite differing 
in HLA-specific pre-disposition, share multiple non-HLA loci, taking part in regulation of the innate and adaptive immunologic responses. Also, both disorders are suspected to be triggered by infections, intestinal dysbiosis and increased intestinal permeability. There are studies highlighting the relationship between genes and intestinal microbiota composition in relation to risk of developing either CD or RA [3].

Recently, it has been confirmed that the spore-forming probiotic strain Bacillus coagulans may have anti-inflammatory and immune-modulating effects in both animals and humans. On the other hand, the prebiotic inulin also potentially influences immunity by changing the gastrointestinal microbiota composition and fermentation profile. Abhari et al. [4] investigated whether $B$. coagulans and inulin, administered either separately or in combination, affect RA activity in rats. Control groups involved a group of rats fed with regular diet and a group receiving indomethacin. Arthritis was induced in rats after 2 weeks of the supplementation or regular diet. Disease activity was evaluated, using biochemica parameters [including fibrinogen, serum amyloid $\mathrm{A}$, tumor necrosis factor-alpha (TNF- $\alpha$ ) and alpha-1-acid glycoprotein levels] as well as paw thickness evaluation. Similarly to the indomethacin group, significant clinical improvement (suppression of paw swelling) as well as a decrease in pro-inflammatory parameters (including fibrinogen, serum amyloid A and TNF- $\alpha$ ) was found in rats pretreated with B. coagulans and/or inulin $(p<0.001)$ [4].

\section{The role of obesity and high-fat diet in rheumatoid arthritis}

Obesity is a civilization disease that is a growing problem, especially in Western countries. It is also a more and more frequent phenomenon in the RA population and carries particular consequences for RA patients. Increased BMI (body mass index) is a known risk factor for developing RA. In a systemic review of 13 studies involving 13,562 RA patients and 400,609 participants in total, it was confirmed that both obesity and overweight increase $\mathrm{RA}$ risk ( $\mathrm{RR}=1.21,95 \% \mathrm{Cl} 1.02-1.44$ and $R R=1.05,95 \% \mathrm{Cl} 0.97-1.13$, respectively). There was a $13 \%$ increase in RA risk for every $5 \mathrm{~kg} / \mathrm{m}^{2}$ increase in BMI. Interestingly, a positive correlation between BMI and RA was found in women and not in men. Among examined subgroups, another significant association between body weight and RA was discovered in the seronegative subgroup $(R R=1.47,95 \% \mathrm{Cl} 1.11-1.96$ for obesity and $R R=1.21,95 \% \mathrm{Cl} 1.06-1.39$ for every $5 \mathrm{~kg} / \mathrm{m}^{2}$ increase in BMI) [5].

There is also evidence that obesity may increase RA activity. One marker of obesity is leptin, a protein pro- duced by adipocytes. In a systematic review involving 13 studies on the relationship between serum leptin levels and rheumatoid arthritis, it was found that plasma leptin level was significantly higher in the RA group than in healthy controls (standardized mean difference $[S M D]=1.056,95 \% \mathrm{Cl}=0.647-1.465, p=4.2 \times 10-7)$, especially in RA patients of Caucasian, Turkish, or Arab origin. There was a statistically significant positive correlation between the serum leptin level and disease activity score (DAS28) (correlation coefficient $=0.275,95 \%$ $\mathrm{Cl}=0.076-0.452, p=0.007$ ), as well as between the serum leptin level and CRP levels (correlation coefficient $=$ $0.274,95 \% \mathrm{Cl}=0.068-0.458, p=0.010$ ) [6].

Furthermore, in a meta-analysis, Liu et al. found evidence that obese patients both obtain and sustain remission less frequently than overweight and/or normal-weight patients (pooled odds ratio [OR] 0.57; 95\% confidence interval $[95 \% \mathrm{Cl}] 0.45,0.72$; pooled OR 0.49; $95 \% \mathrm{Cl} 0.32,0.74$, respectively). They also found that obesity was associated with higher DAS28, tender joint counts, inflammatory marker levels, patient global evaluation and pain scores, as well as physical function scores. However, it was not associated with increased mortality [7].

As for factors leading to obesity, it has been proven that a high-fat diet (HFD) has an adverse impact on arthritis. In a study carried out on mice, the HFD was found to accelerate the development of collagen-induced arthritis (CIA). This effect was connected with higher MIP2/interleukin-2 (IL-2) levels in obese mice. There was no effect of the diet on disease activity. However, compared with obese mice, in mice fed with a normal diet a faster resolution of arthritis was observed. It is suspected that HFD may prolong the inflammatory process induced by the toll-like receptor 4 (TLR-4) by increasing monocytes' migration to inflamed joints and their adipokine-mediated transformation to M1 macrophages a process that is blocked in the case of TLR-4 deficiency or inhibition [8].

As the high-fat diet (HFD) turned out to be a RA risk factor, in order to understand the relationship between HFD and inflammation, Na et al. [9] analyzed T cell parameters in a study on mice with a collagen-induced arthritis (CIA) on HFD. Compared to mice fed with a normal-fat diet, in mice on HFD with a higher metabolic index, the number of inflammation-related Th1 and Th17 cells, among M1 macrophages, was higher. Another important observation concerned zinc-alpha-2-glycoprotein (Azgp1), stimulating lipolysis. Azgp1 level was higher in obese mice. Considering the fact that Azgp1 increased the Th17 population of splenocytes, the authors hypothesized that in RA with coexisting metabolic syndrome, the fat loss acceleration caused by Azgp1 is connected with 
higher IL-17 levels. In fact, mice overexpressing Azgp1 showed more severe CIA compared with other mice [9].

\section{Other potential dietary risk factors for developing rheumatoid arthritis}

In a cross-sectional study involving 1209 adults aged 20-30, it was found that participants consuming sugary drinks rich in fructose such as high-fructose corn syrup, sweetened soft drinks, fruit drinks and apple juice at least 5 times a week had a three times higher risk of developing arthritis (OR $=3.01$; 95\% Cl 1.20-7.59, $p=0.021$ ), irrespective of other dietary factors, plasma glucose levels, physical activity or smoking [10].

In the case of alcohol, surprisingly, no significant correlation between alcohol consumption and RA was discovered in the Västerbotten Intervention Program cohort, which included 386 RA patients, matched to 1,886 healthy controls. Moreover, in this study no significant relationship between diet, evaluated as dietary groups, macronutrients, the carbohydrate-restricted diet score, the Mediterranean diet score and the healthy diet indicator score, and RA was found. However, the authors allow that the results might have been influenced by low alcohol consumption in the studied group overall and other methodological issues [11].

\section{Rheumatoid arthritis prevention through dietary intervention}

In a big prospective study, 76597 women in the Nurses' Health Study aged 30-55 years and 93392 women in the Nurses' Health Study II aged 25-42 years at baseline and free from RA were followed up in order to establish the relationship between healthy eating patterns, as measured by the Alternative Healthy Eating Index 2010 (AHEI-2010), and RA risk. During the course of the study, 1007 new cases of RA were diagnosed. In women aged $\leq 55$ years, better quality diet was associated with a lower RA risk (HRQ4 vs. Q1: 0.67; 95\% Cl 0.51 to 0.88; $p$ trend: 0.002). This association was the strongest for seropositive RA (HRQ4 vs. Q1: 0.60; 95\% Cl 0.42 to 0.86; $p$ trend: 0.003). No significant relationship was found for women aged $>55$ years [12].

Moreover, there are single dietary factors which have been proven to prevent RA. Foods with a preventive action include mushrooms (OR =0.669; 95\% Cl 0.518-0.864; $p=0.002)$, citruses $(\mathrm{OR}=0.990 ; 95 \% \mathrm{Cl} 0.981-0.999$; $p=0.04)$, and dairy $(\mathrm{OR}=0.921 ; 95 \% \mathrm{Cl} 0.867-0.977$; $p=0.006$ ) [13]. In a study from Pattison et al. [14] it was confirmed that daily consumption of a glass of freshly squeezed orange juice is inversely correlated with the risk of RA, probably due to the protective action of beta-cryptoxanthin, a natural carotenoid.

Also, there has been some evidence that high plasma n-3 PUFAs levels lower the risk of RA occurrence in a seropositive population. In a clinical study of 35 seropositive adults with no previous RA history, monitored every 6 months over the period 2008-2014, RA was diagnosed in 14 participants. It was shown that in the studied group a high n-3 PUFAs plasma level was associated with a significantly lower risk of developing arthritis (hazard ratio $[H R]=0.52 ; 95 \% \mathrm{Cl} 0.27-0.98)$ [15]. Factors either promoting or preventing RA are listed in Table I.

\section{The beneficial role of $n-3$ PUFAs in rheumatoid arthritis}

As is commonly known, arachidonic acid, which is a $n-6$ polyunsaturated acid, and prostaglandins deriving directly from the arachidonic acid are associated with pain in the inflammatory process. While n-6 PUFAs have a predominantly pro-inflammatory effect, n-3 PUFAs seem to have anti-inflammatory action. Supplementation with n-3 PUFAs (such as eicosapentaenoic acid and docosahexaenoic acid) has been shown to change favorably the $n-6 / n-3$ fatty acids ratio, reduce inflammation, and alleviate pain, as well as lowering disease activity in rheumatoid arthritis patients. In a systematic review concerning the effect of $n-3$ PUFAs on pain in patients with RA, results from 18 randomized clinical trials involving 1143 patients between 1985 and 2013 were in-

Table I. Dietary factors' influence on rheumatoid arthritis (RA) incidence

\begin{tabular}{|c|c|}
\hline Factors preventing RA & Factors promoting RA \\
\hline $\begin{array}{l}\text { - healthy eating patterns (measured by the Alternative } \\
\text { - Healthy Eating Index 2010) } \\
\text { - } \text { - mushrooms } \\
\text { - citruses, e.g. freshly squeezed orange juice } \\
\text { - dairy }\end{array}$ & $\begin{array}{l}\text { - obesity } \\
\text { - sugary drinks rich in fructose (such as high-fructose corn } \\
\text { - pyrup, sweetened soft drinks, fruit drinks and apple juice) } \\
\text { - viruses (EBNA-1), bacteria (Micrococcus luteus, Mycobacteri- } \\
\text { um gastri, Nocardia brasiliensis), fungi (Aspergillus fumiga- } \\
\text { tus, Candida albicans, Cryptococcus neoformans) } \\
\text { and plants (Oryza sativa, Solanum lycopersicum, Glycine } \\
\text { max) in population colonized with Porphyromonas gingivalis }\end{array}$ \\
\hline
\end{tabular}


cluded. The n-3 PUFAs at a dose of 2.1 up to $9.1 \mathrm{~g} /$ day were administered for 3 months up to 1 year. Pain was assessed either by patients or doctors or both patients and doctors. Out of 18 studies, 10 confirmed pain reduction in patients receiving $n-3$ PUFAs, most of them supporting a daily n-3 PUFAs intake of 3 to $6 \mathrm{~g}$ [16]. Also in a systematic review from Senftleber et al. [17], including 22 randomized trials on marine oils' use in RA, a significant pain relieving effect was found (SMD $-0.21 ; 95 \% \mathrm{Cl}$ -0.42 to -0.004$)$.

In a cross-sectional study using data derived from the Evaluation of Subclinical Cardiovascular Disease and Predictors of Events in RA (ESCAPE-RA) cohort study, the authors explored the relationship between fish consumption frequency in the past year and disease activity. Patients consuming fish more than twice a week had a significantly lower DAS28-CRP compared to subjects consuming fish never to less than once a month [difference -0.49 (95\% Cl $-0.97,-0.02)$ ]. DAS28-CRP was significantly reduced by 0.18 for each additional fish serving per week $(95 \% \mathrm{Cl}-0.35,-0.004)[18]$.

In a prospective, randomized study including $60 \mathrm{pa}$ tients with active rheumatoid arthritis, in the course of 12-week supplementation with fish oil containing n-3 PUFA (group I), fish oil and additionally primrose evening oil, containing $\gamma$-linolenic acid (group II), or with no supplementation (group III), the disease activity as measured by DAS28 was evaluated at week 0 and week 12. At the end of the study, the DAS28 significantly decreased in groups I and II $(p<0.001)$. The plasma $n-6 / n-3$ fatty acid ratio declined from $15.47 \pm 5.51$ to $10.62 \pm 5.07$ $(p=0.005)$ in group I, and from $18.15 \pm 5.04$ to $13.50 \pm 4.81$ $(p=0.005)$ in group II. It was therefore concluded that daily $n-3$ fatty supplementation, either alone or with $\gamma$-linolenic acid, has beneficial effects in lowering the disease activity [19].

In another systemic review including studies published until March 2017, it was found that both in human and animal studies, n-3 PUFAs supplementation not only prevented RA in subjects at risk, but also alleviated the pain, lowered the tender joint count, shortened the morning stiffness duration and reduced the frequency of NSAID use in RA patients. However, there is still a need to investigate the effect of $n-3$ PUFAs on radiographic changes and synovial histopathologic findings, as well as their therapeutic potential both in early arthritis and in combination with biologics [20].

The last question was partly answered in a clinical study involving 22 RA patients treated with TNF- $\alpha$ inhibitors, in which an inverse correlation between plasma eicosapentaenoic acid levels (EPA), as well as plasma EPA/arachidonic acid ratio, and DAS28 after three months of therapy $(-0.51, p=0.007$, and -0.48 , $p=0.01$, respectively) was detected. Additionally, a relationship between serum EPA levels and good treatment response, as defined by EULAR, was found $(p=0.02)$. It may be explained by the fact that while Th17 stimulation was found both to be exaggerated by etanercept in vitro and also to be present in patients with a poor treatment response, EPA intake seems to prevent it [21].

The beneficial role of n-3 PUFAs in lowering disease activity in RA patients may be explained by their action on pro-inflammatory cytokines. In a meta-analysis of 18 randomized clinical trials including 826 RA patients supplemented with n-3 PUFAs, lowered B4 leukotriene levels in RA patients and decreased B2 thromboxane levels in patients from high cardiovascular risk group were observed [22].

\section{Other dietary factors potentially lowering rheumatoid arthritis activity}

In a survey, $24 \%$ of patients reported perceived effects of their diet on RA symptoms; 19\% observed negative effects of sugary drinks and sweets, while 15\% reported beneficial effects of some foods' consumption, most often berries or spinach [23]. It might be worthwhile to try to provide some insight through clinical studies of whether perceptions match reality.

Both low-sodium and low-magnesium diets seem to have some anti-inflammatory potential. In a clinical study, Scrivo et al. [24] investigated links between sodium intake and both the frequency and function of Thelper 17 (Th17) and regulatory T cells (Treg). The study included RA patients known not to be receiving drugs increasing urinary sodium excretion. They first underwent 3 weeks of a low-sodium dietary regimen and then 2 weeks of a normal-sodium dietary regimen. Patients' adherence to regimens was good, as confirmed by urinary sodium excretion analysis. In the RA group, a trend in the Th17 cell frequency reduction on the low-sodium diet and their increase on normal-sodium diet was observed, whereas Treg cells showed the opposite trend. A decrease in TGF $\beta$ and IL-9 serum levels was observed at the endpoint $(p=0.0016$ and $p=0.0007$, respectively). The authors concluded that a low-sodium dietary intake may lower the inflammatory response in RA patients.

Furthermore, in a study performed on rats with pristane-induced arthritis (PIA), the effects of a low-magnesium diet on RA activity and joint damage were investigated. Rats were divided into three groups with different diet regimens: a low-magnesium diet, a normal diet, and a magnesium-supplemented diet. Then after 2 weeks of PIA induction all rats were fed a regular diet. Rats that were initially on the low-magnesium diet 
Table II. Dietary factors' influence on rheumatoid arthritis (RA) activity

\begin{tabular}{|ll|}
\hline \multicolumn{1}{|c|}{ Factors lowering RA activity } & \multicolumn{1}{c|}{ Factors increasing RA activity } \\
\hline - Bacillus coagulans and/or inulin & • obesity \\
- fish/marine oils rich in N-3 PUFAs (eicosapentaenoic and docosahexaenoic & • high-fat diet \\
- moid) alone or together with $\gamma$-linolenic acid and/or with vitamin D & • sugary drinks and sweets (observational) \\
- Mediterranean diet & \\
- low-sodium diet & \\
- low-magnesium diet & \\
- high-methionine diet & \\
- Selenplus capsules \\
- fruit, vegetables and spices rich in polyphenols (Table III) \\
- berries and spinach (observational)
\end{tabular}

had a 70\% lower median arthritis severity score and normal joint histology results. On the other hand, rats remaining initially on a regular or magnesium-supplemented diet developed equally severe disease activity and erosive joint changes. Interestingly, the beneficial effects of low-magnesium diet used in the short term seemed to be lasting. Pathophysiologically, it was observed that rats receiving a short-term low-magnesium diet had significant reduction in synovial gene expression of IL-6, RORA and RORC, responsible for the development of Th17 cells [25].

Other promising data concern a high-methionine (high-Met) diet. In a recent study Li et al. [26] tried to determine whether a high-Met diet may reduce RA adverse effects, such as the loss of body mass. Forty rats with autoimmune arthritis were randomly assigned to a highMet group or to a group on a regular diet. Compared to the control group, in the group on a high-Met diet, an increase in food intake, as well as in body mass, was observed $(p<0.05)$. The high-Met diet seemed to cause suppression of atrogin-1 and MuRF1 gene expression in the gastrocnemius, but not in the soleus. It also caused an increase in IGF-I and a decrease in IGFBP-3 gene expression in both the gastrocnemius and the soleus. The results suggest that arthritis severity may be alleviated by a high-Met diet [26].

The hypothesized RA activity lowering effect by vitamin D has not found confirmation in previous studies. In a systematic review exploring vitamin D serum levels' effect on the development and activity of RA, which included 5 randomized trials on RA patients supplemented with vitamin $D$ analogs for at least 3 months, no difference in disease activity as measured by DAS28 between the group supplemented with vitamin $D$ and the group with no such supplementation was found. However, the recurrence of flares was decreased in patients receiving vitamin D, although the difference was not statistically significant [27]. On the other hand, some data encour- aging vitamin D supplementation in RA patients comes from a Swedish prospective study involving 727 patients with the established diagnosis of early arthritis. It was found that a high vitamin D and n-3 PUFA intake in the time window preceding the beginning of therapy may be associated with a better response to treatment in the first year of therapy [28]. Factors influencing RA activity are presented in Table II.

\section{Antioxidants and rheumatoid arthritis}

Data on antioxidants' role in RA are scarce and often contradictory. It has been speculated that antioxidants improve the healthy immune response and therefore supplementation with microelements such as zinc and selenium, as well as vitamin A, C and E, otherwise known for protecting cell membranes against oxidative stress, has been studied in numerous studies on RA patients. The results were mostly inconclusive or pointed slightly towards their beneficial effects.

In an Iranian pre-post clinical trial, 39 female patients with a mild to moderate severity of RA were supplemented for 12 weeks daily with a Selenplus capsule (Eurovital, Germany) containing 50 mg of selenium, $8 \mathrm{mg}$ of zinc, $400 \mu \mathrm{g}$ of vitamin A, $125 \mathrm{mg}$ of vitamin C, and $40 \mathrm{mg}$ of vitamin E. A significant reduction in serum hs-CRP and DAS-28 score was found ( $p<0.01)$. Similar results were observed by Koracevic et al. [29], who additionally used eicosapentaenoic, docosanoic acid and gamma linolenic acids. However, in both studies, no statistically significant improvement in clinical such as number of swollen or painful joints was found.

The reports on the correlation between serum selenium (Se) levels and rheumatoid arthritis are conflicting. In a meta-analysis including 14 case-control studies, it was observed that RA patients had lower Se serum levels $(\mathrm{SMD}=-1.347,95 \% \mathrm{Cl}-1.872,-0.823, p<0.001)$. However, the statistically significant difference concerned RA patients from Europe and Asia but not from the USA [30]. 


\section{The benefits of polyphenols' consumption in rheumatoid arthritis}

A lot of promising evidence concerns the beneficial anti-oxidative effects of plant polyphenols, contained in fresh fruits, vegetables, and spices, in RA patients. An example of a dietary element rich in polyphenols which may be of benefit in RA is the dried plum. In a study on transgenic mice overexpressing TNF, designed as a RA genetic model, the effects of dried plum (DP) on joint destruction were evaluated. Month-long supplementation with DP not only slowed the onset of arthritis, but also reduced joints' bone erosions compared to mice on a regular diet. Known beneficial effects of DP components, polyphenols, and neochlorogenic acid at the molecular level include the inhibition of TNF-induced formation of osteoclasts. The favorable effect of DP may also be due to lowering the number of tartrate-resistant acid phosphatase (TRAP) positive cells, responsible for osteoclastogenesis [31].

Also a potential anti-inflammatory effect of equol in arthritis was studied. Equol is a major soybean isoflavone metabolite. In a study on mice with collagen-induced arthritis (CIA), the effects of equol on inflammatory response and bone erosion were investigated. Equol was found to both alleviate the severity of arthritis symptoms and slow down the CIA-induced bone mineral density decline. Its immunomodulatory action caused suppression of the expression of IL-6 and its receptor locally in inflamed tissues. Equol also impacted bone metabolism by decreasing the expression of genes associated with inhibition of bone formation, osteoclast and immature osteoblast specificity, as well as cartilage destruction [32].

Another study focused on polyphenol-rich extra virgin olive oil (EVOO), providing evidence of the beneficial effects of EVOO and its minor components in

Table III. Fruit, vegetables, spices and other products rich in polyphenols, lowering levels of pro-inflammatory cytokines connected with rheumatoid arthritis [41]

\begin{tabular}{|lll|}
\hline \multicolumn{1}{|c}{ Fruit and vegetables } & \multicolumn{1}{c}{ Spices } & \multicolumn{1}{c|}{ Other } \\
\hline - dried plum & - black pepper & • wheat, oats, \\
- red grape skin (resver- & - ginger & and maize \\
atrol)-mangoes (man- & - allspice & (p-coumaric \\
giferin) & - caraway & acid) \\
- grapefruits (kaempferol) & - bay leaves & - cocoa \\
- grapes, apples, oranges, & - cinnamon & - rooibos tea \\
spinach, tomatoes, pota- & - licorice & - green tea \\
toes (p-coumaric acid) & - paprika & \\
- soybeans (genistein) & - clove & \\
& - nutmeg & \\
& - chili pepper & \\
& - bilberry & \\
\hline
\end{tabular}

immune-inflammatory diseases including not only rheumatoid arthritis, but also systemic lupus erythematosus, sclerosis and inflammatory bowel disease [33].

In a study on CIA mice, the green tea polyphenol epigallocatechin-3-gallate (EGCG) was found to attenuate arthritis symptoms. At a molecular level, it inhibited osteoclastogenesis and Thelper 17 cell activation, and also increased the regulatory $T$ cell number [34].

Results of a meta-analysis of randomized clinical trials (RCTs) concerning turmeric extracts containing curcumin in patients with arthritis suggested that turmeric extract (about $1000 \mathrm{mg} /$ day of curcumin) alleviated pain symptoms connected with arthritis [35]. Moreover, in a study by Zheng et al. [36], curcumin was shown to decrease the expression of NF- $\kappa B$, TNF- $\alpha$, and IL- $1 \beta$ in the synovial fluid and blood serum. Since the observed effects were similar to effects observed in a standard regimen with methotrexate, the authors of the study speculated that curcumin could be used as anti-rheumatic therapy either in monotherapy or as an adjuvant in RA patients.

Moreover, in a study on Wistar rats, ginger, rich in phenolic compounds such as shogaols and gingerols, as well as turmeric, rich in phenolic curcuminoids, independently had a significant $(p<0.05)$ protective effect against RA [37]. When used together, both compounds had a significant protective effect against extra-articular (hematological, metabolic and cardiovascular) complications of RA [38].

In a study including RA patients receiving 2 capsules of $250 \mathrm{mg}$ of pomegranate extract (POMx) for 8 weeks, a significant reduction in DAS28 $(p<0.001)$, connected with a decrease in swollen and tender joints, pain intensity and erythrocyte sedimentation rate (ESR) levels, was observed in comparison to the control group. Also parameters such as Health Assessment Questionnaire (HAQ) score $(p=0.007)$ and morning stiffness $(p=0.04)$ were significantly improved [39]. Similar results were obtained in a randomized, double-blind, placebo-controlled clinical trial, in which RA patients receiving quercetin (500 mg/day) for 8 weeks had significant improvements in clinical symptoms, including morning stiffness, pain and number of swollen joints, reduction in DAS-28 and HAQ, as well as lower hsTNF- $\alpha$ levels [40].

Studies on polyphenols' impact on lowering levels of pro-inflammatory cytokines taking part in the pathogenesis of RA are numerous and were summarized in a recent review by Islam et al. (data presented in Table III) [41]. Also several herbs have been shown to lower pro-inflammatory cytokines and/or reduce RA clinical symptoms, especially when added to the standard treatment. These medicinal plants have long been used in traditional medicine in the treatment of arthritis. The 
Table IV. Herbs (and their active phytochemicals) lowering pro-inflammatory cytokines levels and potentially reducing rheumatoid arthritis clinical symptoms [42]

\begin{tabular}{|ll|}
\hline Herb & Active phytochemicals \\
\hline Arnica montana & phenolic and flavonoid compounds \\
\hline Boswellia serrata & $\begin{array}{l}\text { a gum resin, known as frankincense and the boswellic acids, a family of pentacy- } \\
\text { clic triterpenes }\end{array}$ \\
\hline Curcuma spp. & $\begin{array}{l}\text { diferuloylmethane, a yellow phenolic pigment commonly known as curcumin, } \\
\text { and } \beta \text {-elemene }\end{array}$ \\
\hline Equisetum arvense (horsetail) & kynurenic acid \\
\hline Harpagophytum procumbens (devil's claw) & iridoid glycosides such as harpagoside, harpagide, and procumbide \\
\hline Panax notoginseng & saponins \\
\hline Salix spp. (willow) & salicin, polyphenols and flavonoids \\
\hline Sesamum indicum & lignans; sesamin and sesamolin degraded to sesamol \\
\hline Symphytum officinale (comfrey) & phenolic acids such as rosmarinic acid, glycopeptides and amino acids \\
\hline Zingiber officinalis (ginger) & polyphenols such as gingerols and shogaols \\
\hline Withania somnifera (ashwagandha) & withaferin A \\
\hline
\end{tabular}

evidence for their use has been summarized in a review from Dragos et al. [42] and collected in Table IV.

\section{Is the Mediterranean diet recommended for rheumatoid arthritis patients?}

The subject of the Mediterranean diet's effect on RA is controversial. Some authors, such as Bloomfield et al., [43] point out that while many studies have confirmed a beneficial role of the Mediterranean diet in preventing cardiovascular events, cancer and diabetes, no such role in RA has been proven. It seems to be confirmed by a prospective study involving initially healthy US female adults: 83,245 participants from the Nurses' Health Study (NHS; 1980-2008) and 91,393 participants from NHS II (1991-2009), in which no significant association between a Mediterranean dietary pattern, assessed using the Alternate Mediterranean Diet Score (aMed), and the risk of RA was found. During the 4-year follow-up, 913 new cases of RA were diagnosed among the participants. After adjustment for other factors, alcohol was the only food component of the aMed score associated with an altered risk of incident RA [44].

On the other hand, in a systematic review of prospective studies exploring the Mediterranean diet's effects on RA prophylaxis and management, in two out of four studies, in patients on the Mediterranean diet, an improvement in pain, assessed by the visual analogue scale $(p<0.05)$, as well as a decrease in the HAQ score $(p<0.05)$, was found. In one study, in the Mediterranean diet group a DAS28 decrease was observed $(p<0.05)$. However, no beneficial effect of the Mediterranean diet on RA prevention was found [45].
In a study involving 208 RA patients, matched to 205 healthy controls from the prospective TOMORROW cohort study, the authors tried to identify components of the Mediterranean diet that may potentially lower disease activity. It was found that DAS28-ESR had a significant correlation with monounsaturated fatty acid/saturated fatty acid (MUFA/SFA) intake after age adjustment $(R=-0.228$, $p<0.01)$. It was also discovered that the MUFA intake was significantly lower in the RA compared to the control group ( $p=0.003$ ). The logistic regression analysis with borderline significance showed high MUFA intake as an independent predictor of remission in the RA group (OR = $1.97 ; 95 \% \mathrm{Cl}=0.98-3.98 ; p=0.057)$. It was therefore concluded that MUFA intake may be the Mediterranean diet component responsible for RA activity suppression [46].

\section{Points to consider}

In a cross sectional study addressing the possible relationship between the diet (assessed by the HEI-2010), RA activity and functional status (assessed using blood tests and the Multidimensional Health Assessment Questionnaire), it was discovered that most patients did not comply with healthy diet recommendations. The mean HEI-2010 total score was $58.7 \pm 15.9$, where $7.1 \%$ of patients achieved a "good", 58.3\% a "fair", and 34.5\% a "poor" result. In an unadjusted multiple linear regression model it was found that the morning stiffness duration and C-reactive protein level were significant variables inversely predicting HEI-2010 total score [47].

The results of the above study indicate that RA patients' adherence to a healthy diet remains low, despite there being more and more data confirming positive ef- 
fects of healthy foods, mostly fish rich in n-3 PUFAs, as well as a negative influence of eating patterns generally considered unhealthy, such as a high-fat diet or high sugary drink and sweet consumption, on RA incidence, activity and treatment response. A great responsibility lies with physicians treating RA patients, in educating both their patients and themselves on the potential benefits from a simple dietary intervention.

The authors declare no conflict of interest.

The rights revenue was paid by Stowarzyszenie Zbiorowego Zarządzania Prawami Autorskimi Twórców Dziet Naukowych i Technicznych KOPIPOL of Kielce from fees collected pursuant to Article 20 and Article $20^{1}$ of the Copyright and Neigbouring Rights Act.

Wynagrodzenie autorskie sfinansowane zostato przez Stowarzyszenie Zbiorowego Zarzadzania Prawami Autorskimi Twórców Dziet Naukowych i Technicznych KOPIPOL z siedziba w Kielcach z opłat uzyskanych na podstawie art. 20 oraz art. $20^{1}$ ustawy o prawie autorskim i prawach pokrewnych.

\section{References}

1. Tsuda R, Ozawa T, Kobayashi E, et al. Monoclonal Antibody Against Citrullinated Peptides Obtained From Rheumatoid Arthritis Patients Reacts With Numerous Citrullinated Microbial and Food Proteins. Arthritis Rheumatol 2015; 67: 2020-2031.

2. Gong T, Wang X, Yang Y, et al. Plant Lectins Activate the NLRP3 Inflammasome To Promote Inflammatory Disorders. J Immunol 2017; 198: 2082-2092.

3. Lerner A, Matthias T. Rheumatoid arthritis-celiac disease relationship: Joints get that gut feeling. Autoimmun Rev 2015; 14: 1038-1047.

4. Abhari K, Shekarforoush SS, Hosseinzadeh S, et al. The effects of orally administered Bacillus coagulans and inulin on prevention and progression of rheumatoid arthritis in rats. Food Nutr Res 2016; 60: 30876.

5. Feng J, Chen Q, Yu F, et al. Body Mass Index and Risk of Rheumatoid Arthritis. Medicine (Baltimore) 2016; 95: e2859.

6. Lee YH, Bae SC. Circulating leptin level in rheumatoid arthritis and its correlation with disease activity: a meta-analysis. Z Rheumatol 2016; 75: 1021-1027.

7. Liu Y, Hazlewood GS, Kaplan GG, et al. Impact of Obesity on Remission and Disease Activity in Rheumatoid Arthritis: A Systematic Review and Meta-Analysis. Arthritis Care Res (Hoboken) 2017; 69: 157-165.

8. Kim S, Chen Z, Essani AB, et al. Differential impact of obesity on the pathogenesis of RA or preclinical models is contingent on the disease status. Ann Rheum Dis 2017; 76: 731-739.

9. Na HS, Kwon J-E, Lee SH, et al. Th17 and IL-17 Cause Acceleration of Inflammation and Fat Loss by Inducing $\alpha$ 2-Glycoprotein 1 (AZGP1) in Rheumatoid Arthritis with High-Fat Diet. Am J Pathol 2017; 187: 1049-1058.

10. DeChristopher LR, Uribarri J, Tucker KL. Intake of high-fructose corn syrup sweetened soft drinks, fruit drinks and apple juice is associated with prevalent arthritis in US adults, aged 20-30 years. Nutr Diabetes 2016; 6: e199.

11. Sundström B, Johansson I, Rantapää-Dahlqvist S. Diet and alcohol as risk factors for rheumatoid arthritis: a nested casecontrol study. Rheumatol Int 2015; 35: 533-539.

12. Hu Y, Sparks JA, Malspeis S, et al. Long-term dietary quality and risk of developing rheumatoid arthritis in women. Ann Rheum Dis 2017; 76: 1357-1364.

13. He J, Wang $Y$, Feng $M$, et al. Dietary intake and risk of rheumatoid arthritis - a cross section multicenter study. Clin Rheumatol 2016; 35: 2901-2908.

14. Pattison DJ, Symmons DPM, Lunt M, et al. Dietary beta-cryptoxanthin and inflammatory polyarthritis: results from a population-based prospective study. Am J Clin Nutr 2005; 82: 451-455.

15. Gan RW, Demoruelle MK, Deane KD, et al. Omega-3 fatty acids are associated with a lower prevalence of autoantibodies in shared epitope-positive subjects at risk for rheumatoid arthritis. Ann Rheum Dis 2017; 76: 147-152.

16. Abdulrazaq M, Innes JK, Calder PC. Effect of $\omega-3$ polyunsaturated fatty acids on arthritic pain: A systematic review. Nutrition 2017; 39-40: 57-66.

17. Senftleber NK, Nielsen SM, Andersen JR, et al. Marine Oil Supplements for Arthritis Pain: A Systematic Review and Meta-Analysis of Randomized Trials. Nutrients 2017; 9. pii: E42

18. Tedeschi SK, Bathon JM, Giles JT, et al. The relationship between fish consumption and disease activity in rheumatoid arthritis. Arthritis Care Res (Hoboken) 2018; 70: 327-332.

19. Veselinovic M, Vasiljevic D, Vucic V, et al. Clinical Benefits of n-3 PUFA and $\gamma$-Linolenic Acid in Patients with Rheumatoid Arthritis. Nutrients 2017; 9: 325.

20. Navarini L, Afeltra A, Gallo Afflitto G, Margiotta DPE. Polyunsaturated fatty acids: any role in rheumatoid arthritis? Lipids Health Dis 2017; 16: 197.

21. Jeffery L, Fisk HL, Calder PC, et al. Plasma Levels of Eicosapentaenoic Acid Are Associated with Anti-TNF Responsiveness in Rheumatoid Arthritis and Inhibit the Etanercept-driven Rise in Th17 Cell Differentiation in Vitro. J Rheumatol 2017; 44: 748756.

22. Jiang J, Li K, Wang F, et al. Effect of Marine-Derived n-3 Polyunsaturated Fatty Acids on Major Eicosanoids: A Systematic Review and Meta-Analysis from 18 Randomized Controlled Trials. Sampson AP, ed. PLoS One 2016; 11: e0147351.

23. Tedeschi SK, Frits M, Cui J, et al. Diet and Rheumatoid Arthritis Symptoms: Survey Results From a Rheumatoid Arthritis Registry. Arthritis Care Res (Hoboken) 2017; 69: 1920-1925.

24. Scrivo R, Massaro L, Barbati C, et al. The role of dietary sodium intake on the modulation of $T$ helper 17 cells and regulatory $T$ cells in patients with rheumatoid arthritis and systemic lupus erythematosus. PLoS One 2017; 12: e0184449.

25. Brenner M, Laragione T, Gulko PS. Short-term low-magnesium diet reduces autoimmune arthritis severity and synovial tissue gene expression. Physiol Genomics 2017; 49: 238-242.

26. Li M, Zhai L, Wei W. High-Methionine Diet Attenuates Severity of Arthritis and Modulates IGF-I Related Gene Expressions in an Adjuvant Arthritis Rats Model. Mediators Inflamm 2016; 2016: 9280529.

27. Franco AS, Freitas TQ, Bernardo WM, Pereiria RMR. Vitamin D supplementation and disease activity in patients with im- 
mune-mediated rheumatic diseases A systematic review and meta-analysis. Medicine (Baltimore) 2017; 96: e7024.

28. Lourdudoss C, Wolk A, Nise L, et al. Are dietary vitamin D, omega-3 fatty acids and folate associated with treatment results in patients with early rheumatoid arthritis? Data from a Swedish population-based prospective study. BMJ Open 2017; 7: e016154.

29. Jalili M, Kolahi S, Aref-Hosseini S-R, et al. Beneficial role of antioxidants on clinical outcomes and erythrocyte antioxidant parameters in rheumatoid arthritis patients. Int J Prev Med 2014; 5: 835-840.

30. Yu N, Han F, Lin X, et al. The Association Between Serum Selenium Levels with Rheumatoid Arthritis. Biol Trace Elem Res 2016; 172: 46-52.

31. Mirza F, Lorenzo J, Drissi H, et al. Dried plum alleviates symptoms of inflammatory arthritis in TNF transgenic mice. J Nutr Biochem 2018; 52: 54-61.

32. Lin I-C, Yamashita S, Murata M, et al. Equol suppresses inflammatory response and bone erosion due to rheumatoid arthritis in mice. J Nutr Biochem 2016; 32: 101-106.

33. Aparicio-Soto M, Sánchez-Hidalgo M, Rosillo MÁ, et al. Extra virgin olive oil: a key functional food for prevention of immune-inflammatory diseases. Food Funct 2016; 7: 4492-4505.

34. Ahmed S. Green tea polyphenol epigallocatechin 3-gallate in arthritis: progress and promise. Arthritis Res Ther 2010; 12: 208.

35. Daily JW, Yang M, Park S. Efficacy of Turmeric Extracts and Curcumin for Alleviating the Symptoms of Joint Arthritis: A Systematic Review and Meta-Analysis of Randomized Clinical Trials. J Med Food 2016; 19: 717-729.

36. Cai $H$, Zheng $Z$, Sun $Y$, et al. The effect of curcumin and its nanoformulation on adjuvant-induced arthritis in rats. Drug Des Devel Ther 2015; 9: 4931.

37. Ramadan G, Al-Kahtani MA, El-Sayed WM. Anti-inflammatory and Anti-oxidant Properties of Curcuma longa (Turmeric) Versus Zingiber officinale (Ginger) Rhizomes in Rat Adjuvant-Induced Arthritis. Inflammation 2011; 34: 291-301.
38. Ramadan G, El-Menshawy O. Protective effects of ginger-turmeric rhizomes mixture on joint inflammation, atherogenesis, kidney dysfunction and other complications in a rat model of human rheumatoid arthritis. Int J Rheum Dis 2013; 16: 219-229.

39. Ghavipour M, Sotoudeh G, Tavakoli E, et al. Pomegranate extract alleviates disease activity and some blood biomarkers of inflammation and oxidative stress in Rheumatoid Arthritis patients. Eur J Clin Nutr 2017; 71: 92-96.

40. Javadi F, Ahmadzadeh A, Eghtesadi S, et al. The Effect of Quercetin on Inflammatory Factors and Clinical Symptoms in Women with Rheumatoid Arthritis: A Double-Blind, Randomized Controlled Trial. J Am Coll Nutr 2017; 36: 9-15.

41. Islam MA, Alam F, Solayman M, et al. Dietary Phytochemicals: Natural Swords Combating Inflammation and Oxidation-Mediated Degenerative Diseases. Oxid Med Cell Longev 2016; 2016: 5137431.

42. Dragos D, Gilca M, Gaman L, et al. Phytomedicine in Joint Disorders. Nutrients 2017; 9. pii: E70.

43. Bloomfield HE, Koeller E, Greer N, et al. Effects on Health Outcomes of a Mediterranean Diet With No Restriction on Fat Intake. Ann Intern Med 2016; 165: 491.

44. Hu Y, Costenbader KH, Gao X, et al Mediterranean diet and incidence of rheumatoid arthritis in women. Arthritis Care Res (Hoboken) 2015; 67: 597-606.

45. Forsyth C, Kouvari M, D'Cunha NM, et al. The effects of the Mediterranean diet on rheumatoid arthritis prevention and treatment: a systematic review of human prospective studies. Rheumatol Int 2018; 38: 737-747.

46. Matsumoto Y, Sugioka Y, Tada M, et al. Monounsaturated fatty acids might be key factors in the Mediterranean diet that suppress rheumatoid arthritis disease activity: The TOMORROW study. Clin Nutr 2018; 37: 675-680.

47. Berube LT, Kiely M, Yazici Y, Woolf K. Diet quality of individuals with rheumatoid arthritis using the Healthy Eating Index (HEI)-2010. Nutr Health 2017; 23: 17-24. 